\title{
EXPLORING VISUAL MEDIA IN THE PROCESS OF ETHNOGRAPHIC FIELDWORK
}

\author{
// KAMILLA NØRTOFT \& ANNE-KATRINE HANSEN
}

\begin{abstract}
This paper discusses the benefits of using film in ethnographic fieldwork. During the fieldwork for Kamilla's Ph.D. project about elderly people's health and social relations, we have experimented with the use of video. In this paper we focus on the results of and reactions to using self-produced film material in focus group discussions conducted in an activity centre for retired people in Copenhagen. The method applied turned out to be beneficial in expected but also positively surprising ways that enabled us to gain insights beyond those of solely word-based approaches.
\end{abstract}

Please view our short film clip Bodil in the Snow (at http://vimeo.com/59180448) which we used for accessing knowledge about health issues among the elderly in Copenhagen, as discussed in the article. 


\section{INTRODUCTION}

$\mathrm{n}$ this methodological paper, we wish to discuss the use of film in ethnographic fieldwork and analysis, briefly touching upon issues of representation. Our collaboration as a visual and a non-visual anthropologist has led to fruitful discussions throughout the process about the place and possibility of film as an academic genre. If by scientific work, we mean contributing to theoretical discussions and academic knowledge about a certain theme, how can we convey this and present the different processes in doing so using film?

As part of Kamilla's Ph.D. project, we experimented with film at different stages of the anthropological research process:

- As a way to gain access to the field and sensory

data about growing old

- To generate observational data

- To use the audiovisual data in analysis

- To convey results of the research

We believe that film can convey knowledge in a manner that speaks directly to the senses, and can convey an embodied knowledge or experience of the world. Furthermore, we wanted an open approach where we would experiment with the possibilities of the medium as the fieldwork progressed and our knowledge improved.

Our research addresses how health and health-related issues are expressed, spoken of, perceived and performed in different institutional settings. The project focuses on healthcare work with elderly people in the municipality of Copenhagen and deals with elderly people and social relations connected to health in a very broad sense.

The main methods used in the field have been participant observation (with and without a camera), formal and informal interviews, and focus group discussions.

The fieldwork took place in an activity centre for elderly people managed by the City of Copenhagen. The purpose of the centre is to provide a platform for social interaction and health activities. The people who attend the centre are retired from work and live in their own homes. Most of them visit the activity centre for socializing, using fitness facilities and taking part in organised activities. From a managerial perspective, the focus is on physical and mental wellbeing in an attempt to address the serious problem of loneliness among elderly citizens in the municipality.

\section{FILM AS A KEY TO ‘OPEN’ THE FIELD}

In recent years, visual anthropology has gained acknowledgement for the use of audiovisual media to capture, express and convey sensory and bodily aspects of informants' life worlds (MacDougall 2006: 59\&268). Anthropological research had originally mainly been language based, but the growing recognition of the fact that not everything can be put into words has given visual (and sensuous) anthropology a lead in the study of these aspects (Ibid: 1ff, Pink 2009: 11). As MacDougall writes, the images of the world we experience through vision do not only become comprehensible to us through a thoughtinduced, language-based abstraction or categorisation, even if it often seems like that. They are included in the construction of our understanding of the world, which is based both on images and experience rather than on abstract knowledge (MacDougall 2006: 3ff).

These views inspired us to use film not only to register and create data and knowledge ourselves, but also to engage our informants in the process of knowledge production by letting them engage with filmic material. In this sense, the use of film facilitated an opening of the field: it broadened our perspective and we gained insights into aspects of the informants' experiences and their situation as elderly people. The notion of one's own body and health is necessarily an embodied experience that can be difficult to put in words. Even though bodily experiences are something we all share and can relate to (Pink 2009: $23 \mathrm{ff}$ ), at our age, we can only imagine the bodily changes and problems that accompany old age. We thus intended to gain access to these notions, and to find a way of opening up these difficult aspects of our field to discussion.

\section{FOCUS GROUP DISCUSSIONS ABOUT BODIL}

We have experimented with accessing such knowledge through focus group discussions, where we gathered a number of informants and showed them film clips to initiate conversation. In the beginning of our fieldwork, we experienced difficulties talking to our interlocutors about health and health-related issues without them consequently referring to measurable factors such as diet, smoking, alcohol consumption and exercise. We ascribe this to the fact that most public preventive health work in Denmark is centred around these so-called 'KRAM factors'.

We decided to produce our own filmic material and let our informants comment on it and discuss what they saw. We wanted to present material about a person our informants could relate to. At the same time, we wanted that person to be someone unknown to them, in order for them to freely comment on what they saw. We decided that Anne-Katrine would follow her grandmother, Bodil, with the camera for three days, and we made seven clips of approximately five minutes each. In each clip, Bodil is either doing something or talking about an issue relevant to our research questions, such as taking her medication, eating, engaging in hobbies, going shopping, and talking about loneliness, ageing and dying.

During the sessions in the activity centre, we proceeded to show one clip and opened the discussion before showing the next one. The informants' interest was evident through their comments and bursts of laughter. Between 


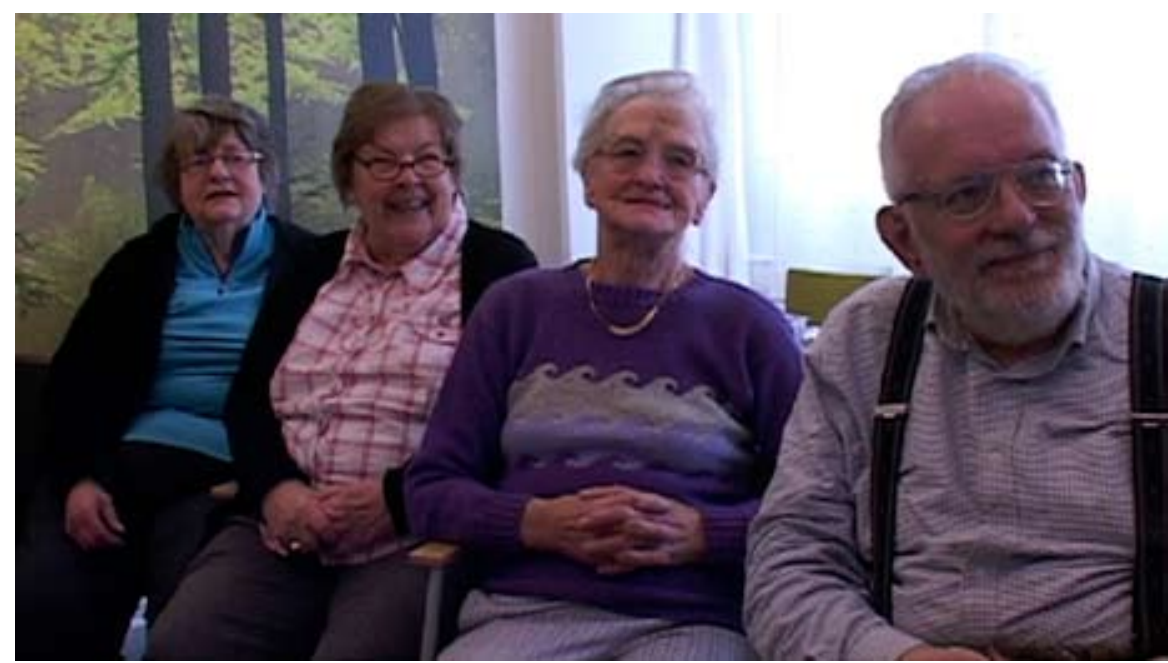

Figure 1: A focus group watching film clips about Bodil

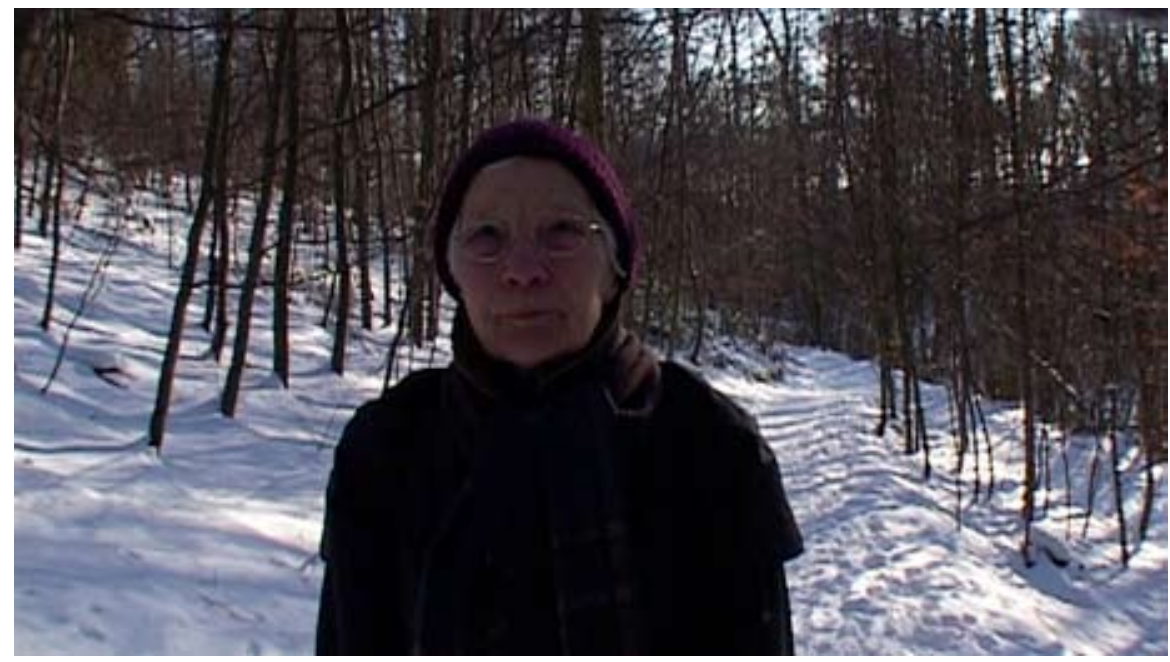

Figure 2: Still of Bodil stopping to catch her breath

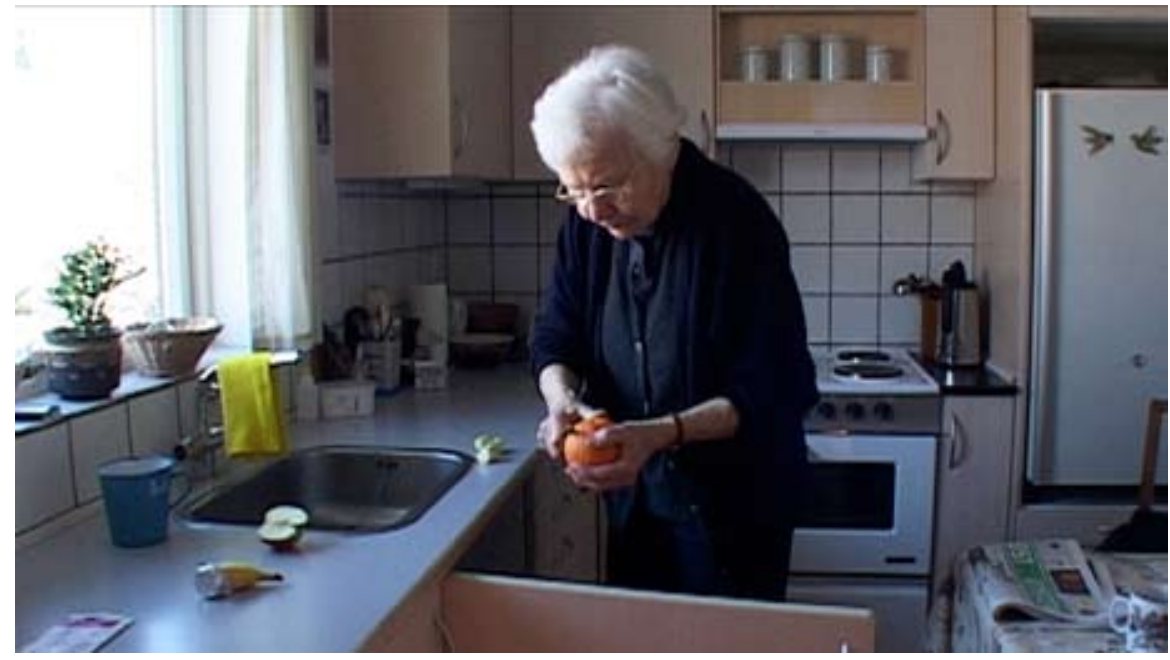

Figure 3: Still of Bodil peeling an orange 
the clips, the informants' comments would be elaborated and clarified and at times more general discussions about a topic would evolve.

\section{Results from filmic material as the basis of discussions}

We could have chosen objects, photos, case stories etc. to represent different themes or topics as the basis of discussions; but while these might also have gotten us beyond the focus on the KRAM factors, our clips of Bodil turned out to give the bodily affiliation or experiential resonance that we sought. Our informants saw Bodil in several daily situations similar to situations in their own daily lives, which resulted in recognition, empathy and a feeling of relatedness between Bodil and the viewers. They felt acquainted with Bodil after seeing her in different situations and talking about different issues relevant to their own lives.

The clips served their purpose in the way we had anticipated: our informants talked about their daily lives in a different manner than when we had chatted with them at the coffee tables in the activity centre. They did not just talk about diet, alcohol, smoking and exercise in a factual manner, but about their lives, everyday situations, and health problems in a much more detailed, experiencebased way.

Bodil in the snow - an example activating a sensory experience

When seeing objects or bodies on film, the knowledge the viewer has of the world creates a sensory interchange that transforms the visual nature of an image into an experience of how it feels (MacDougall 1998: 51). Thus, a more experienced understanding of the informants' worlds is created when viewing the film. MacDougall further argues that film brings back a focus on physical objects, thus emphasizing the human environment, or 'social landscape', which he defines as: 'a distinctive sensory complex, constructed not only of material things but also of human activities and the bodies of human beings themselves' (MacDougall 2006: 58). These sensory aspects are examples of the advantage and quality of using film as method and representation within anthropology, providing

...strategies for exploring dimensions of social life different from those already defined in verbal and quantitative terms. If we ask what aspects of culture are specifically accessible to such an approach, it is often mistakably assumed that it is those that are visible. It is therefore important to assert at once that the visible are equally a pathway to the non-visible, and to the larger domain of the feelings, the intellect and the remaining senses (MacDougall 2006: 269).

When we showed our informants the film clips of Bodil, we experienced this haptic quality of vision. The infor- mants sensed and commented on issues that we had not noticed or thought about because we did not have a bodily experience of them and, therefore, had no frames of reference to them. For example, in the clip where Bodil walks through a forest in the snow to get to the supermarket, we saw an old, yet strong, woman who adjusted her daily activities to her functional level and health condition. However, our informants saw a peer walking quite fast with no support through a hilly forest covered in snow. That she needed no support was their main focus point. A long, cold winter would have kept many of them inside their houses if they had to walk through a forest without any help. Our informants already had bodily experiences of getting old and being weaker than in their younger days, and they had daily experiences of scaling pavements covered with snow and ice in that condition. Therefore, they related to Bodil's rigours in a different way than we could.

We could have asked them how they were dealing with icy pavements and the like, and they would probably have answered that the snow and ice restrained their activities outside the house, or that they felt unsafe and were afraid of falling. However, instead of talking about what was hard for them, they talked about how well Bodil was walking and managing other daily tasks. To see Bodil moving and straining created resonance in our informants and brought out feelings and memories from their own experiences of walking in snow. Furthermore, their bodily reactions were visible to us while they watched: when Bodil walks uphill and stops, out of breath, the informants responded by also breathing in deeply. Such reactions made their experiences clear to us in a very concrete manner as compared to when they told us about the same things (rather than doing them with their bodies).

\section{Long term effects of the 'opening' of the field}

Bodil and the film clips became a common frame of reference between our informants and us in the longer run. After seeing the clips, our interlocutors would often refer to them during our conversations at the coffee tables. It was obvious that our informants felt that they knew Bodil in some way, and would talk about how she did different things. But they did not actually know her in person and, thus, it seemed completely appropriate to talk about her and discuss her in connection to various topics: it did not compromise any social relations but gave them instead a new topic for their daily chats.

The film about Bodil thus helped in creating a rapport in the field as well as in making the informants create different kinds of descriptions of their daily lives than previously experienced. They no longer talked about the concept of health framed within the Danish official discourse of the KRAM factors, but instead about their own experiences. 
DISCUSSION:

DIFFERENT WAYS OF USING FILM AS A METHOD

The themes of health and everyday experiences that we tried to bring into focus in the clips with Bodil definitely came across and evoked the bodily resemblance in the informants that we had hoped for, giving us a good point of departure for understanding what health meant to our informants. As we have illustrated, using film as a means to open the field can be quite productive, since film can communicate on a non-verbal level, bringing into play factors that did not arise when we only addressed the topic verbally in the initial talks with our informants.

Showing the clips proved to be a fruitful method to gain sensory insight as well as bringing us closer to our informants. One reason that it worked so well might be that seeing the clips was a positive shared experience for our informants (and us). It also made our purpose and research questions clearer to our informants.. It was no longer about us trying to 'gain' something from them we also positively contributed in a very tangible way to life in the activity centre.

\section{A note on analysis}

In comparison to the textual conveyance of an analysis, the film-based representation of an argument still may not be so simple. If analytical points are not explained with words during a filmic representation, the final analysis is left with the viewer. Some will consider this a weakness while others might see it as strength (Mermin 1997: 43).

We think it can be both. Not being told which points to focus on in the presented material allows the viewers to focus on the specific point(s) they find most relevant and interesting. It is a weakness in the sense that the anthropologist might not get his or her message across to the viewers and be unable to lead the viewers' attention to something which might be new to them.

This issue of conveying the analysis is not easily bought within the frames of anthropology, traditionally 'oriented to writing' (Marcus 1994: 37). If you cannot follow (see) the argument, how can you judge its validity? It also touches upon the ever-present anthropological problem of representation and authorship taken up by George Marcus and James Clifford's 1986 Writing Culture and the debate that followed. Who is entitled to represent whom, why and how? What about the problem of hegemony and power relations in representations, which are accepted as truth (Ruby 1991: 53)? And, citing the example of the viewers' reactions to Bodil in the snow, whose truth is it: that of the anthropologist, the informants or the viewers?

This raises the question about the visibility of analysis through a theoretical lens. Does it make sense to insist on this visibility when we are not able to direct or control the reception anyway? The material, of course, has a very high degree of autonomous life, living in a dialectic rela- tionship between our selection and presentation of material and the receivers' experiences and attention.

Interestingly, almost regardless of the way we present the film clips with words accentuating our analytical points, the viewers still see and interpret them differently, depending on their perspective and their own experiences. This is, of course, not only the case for filmic material, but the process of showing the clips while we were together with our audience has made the point very clear. This can be illustrated with the clip in which Bodil peels an orange and afterwards eats it while doing a crossword puzzle. Ergonomists have noted how strong Bodil's hands and fingers are compared to those of her age. Dieticians have noted that she is skinny, and that she needs to supplement the healthy orange with foods that contain more calories. Our focus was that she eats healthy food, but also that she eats alone, having the crossword puzzle as her dinner company - a fact our informants also pointed out.

\section{CONCLUSION: AN INVITATION TO CONTINUE THE DISCUSSION}

The project has confirmed that the use of film in the research process has something distinctive to offer. Addressing our research questions by using film clips to evoke bodily experiences provided access to insights we could not have thought of or asked about otherwise. Being younger than our interlocutors, we have not had these experiences ourselves. The film clips made our informants talk about and show their own bodily experiences in ways that went beyond references to public health messages. The film clips also provided an effective way of connecting to our informants by creating a common ground to talk from. Not only did it give an entrance point for discussions, it also gave them an understanding of our interests and of why we were there. An additional benefit was the positive contribution to the daily life in the activity centre.

Using film has been fruitful in accessing knowledge, while at the same time calling into question how this knowledge may be communicated. Is it possible to convey an analysis only as visual representation? Is it a requirement to use words to explain an analysis in order to represent it? These are questions that visual anthropologists are constantly struggling with when debating with less visually oriented colleagues and among themselves (Pink 2009: 119ff). Our non-visual and visual background makes us constantly engage with these discussions as well, and these are questions that we are still struggling with in our work.

Presentations of our material for different audiences have indicated the benefits of combining visual and non-visual representation. The different qualities of visual and nonvisual representational approaches underline how words and motion pictures create different reactions and kinds of knowledge, which do not exclude each other and can- 
not be evaluated with equal measures. We thus agree that anthropological film has to be dealt with on its own terms. It does not make sense to judge it using the same criteria used to evaluate academic texts.

As a conclusion to this paper and an invitation to continue the discussion, we will pass on a question we were asked by a philosopher and former colleague of ours: is it possible, in a meaningful way, to answer and discuss a conveyed message in a different medium than it was originally conveyed in? Should film be answered with film, text with text, music with music and so on? Perhaps there is no one answer to this, but we think it is a good question to tickle the mind and engage in our understanding of academic communication. 佔

\section{ACKNOWLEDGEMENTS}

The production of the audiovisual material presented in this article was financed by the EGV Foundation.
REFERENCES

Clifford, J. \& Marcus, G. E. (1986) Writing Culture. The Poetics and Politics of Ethnography. Berkeley, Los Angeles, London: University of California Press.

MacDougall, D. (1998) Transcultural Cinema. Princeton: Princeton University Press.

MacDougall, D. (2006) The Corporeal Image: Film, Ethnography, and the Senses. Princeton, Woodstock: Princeton University Press.

Marcus, G. E. (1994 ) 'The Modernist Sensibility in Recent Ethnographic Writing and the Cinematic Metaphor of Montage'. In: Taylor, L. (ed.): Visualizing Theory. Selected Essays from V.A.R. 1990-1994, pp. 37-53. London: Routledge.

Mermin, E. (1997) 'Being Where? Experiencing Narratives of Ethnographic Film'. In: Visual Anthropology Review. 13 (1): 40-52.

Pink, S. (2009) Doing Visual Ethnography. London: Sage Publications Ltd.

Ruby, J. (1991) 'Speaking For, Speaking About, Speaking With, or Speaking Alongside - An Anthropological and Documentary Dilemma'. In: Visual Anthropology Review. 7 (2): 50-67. 